A second order accurate embedded boundary method for the wave equation with Dirichlet data

H. O. Kreiss, N. A. Petersson

March 3, 2004

SIAM Journal on Scientific Computing 
This document was prepared as an account of work sponsored by an agency of the United States Government. Neither the United States Government nor the University of California nor any of their employees, makes any warranty, express or implied, or assumes any legal liability or responsibility for the accuracy, completeness, or usefulness of any information, apparatus, product, or process disclosed, or represents that its use would not infringe privately owned rights. Reference herein to any specific commercial product, process, or service by trade name, trademark, manufacturer, or otherwise, does not necessarily constitute or imply its endorsement, recommendation, or favoring by the United States Government or the University of California. The views and opinions of authors expressed herein do not necessarily state or reflect those of the United States Government or the University of California, and shall not be used for advertising or product endorsement purposes. 


\title{
A second order accurate embedded boundary method for the wave equation with Dirichlet data*
}

\author{
Heinz-Otto Kreiss ${ }^{\dagger} \quad$ N. Anders Petersson ${ }^{\ddagger}$
}

March 2, 2004

\begin{abstract}
The accuracy of Cartesian embedded boundary methods for the second order wave equation in general two-dimensional domains subject to Dirichlet boundary conditions is analyzed. Based on the analysis, we develop a numerical method where both the solution and its gradient are second order accurate. We avoid the small-cell stiffness problem without sacrificing the second order accuracy by adding a small artificial term to the Dirichlet boundary condition. Long-time stability of the method is obtained by adding a small fourth order dissipative term. Several numerical examples are provided to demonstrate the accuracy and stability of the method. The method is also used to solve the two-dimensional $\mathrm{TM}_{z}$ problem for Maxwell's equations posed as a second order wave equation for the electric field coupled to ordinary differential equations for the magnetic field.
\end{abstract}

\section{Introduction}

Consider the Dirichlet problem for the second order wave equation in a two-dimensional domain $\Omega$ with boundary $\Gamma$,

$$
\begin{aligned}
& u_{t t}=\Delta u+F(\boldsymbol{x}, t), \quad \boldsymbol{x} \in \Omega, \quad t \geq 0, \\
& u(\boldsymbol{x}, t)=f(\boldsymbol{x}, t), \quad \boldsymbol{x} \in \Gamma, \quad t \geq 0, \\
& u(\boldsymbol{x}, 0)=u_{0}(\boldsymbol{x}), \quad \boldsymbol{x} \in \Omega, \\
& u_{t}(\boldsymbol{x}, 0)=u_{1}(\boldsymbol{x}), \quad \boldsymbol{x} \in \Omega .
\end{aligned}
$$

*This work was performed under the auspices of the U.S. Department of Energy by University of California Lawrence Livermore National Laboratory under contract No. W-7405-Eng-48.

${ }^{\dagger}$ Department of Mathematics, University of California Los Angeles, CA 90024.

${ }^{\ddagger}$ Center for Applied Scientific Computing, Lawrence Livermore National Lab, Livermore, CA 94551, andersp@llnl.gov. 


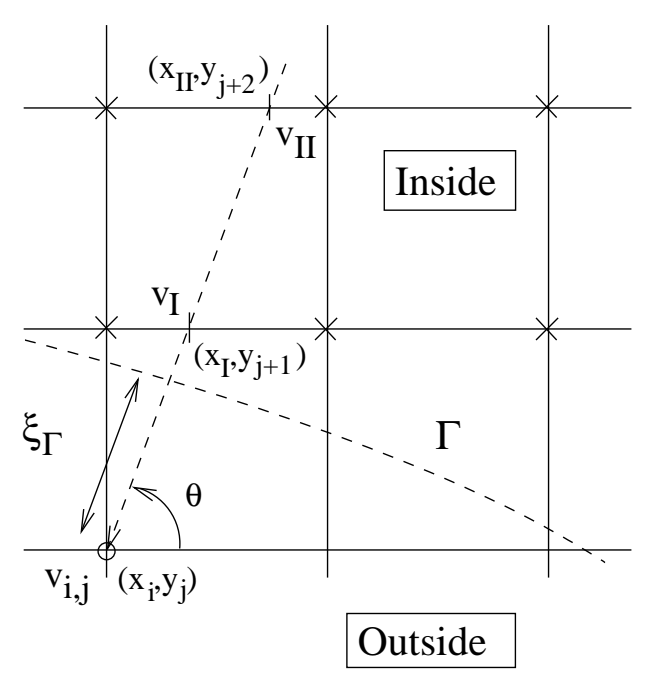

Figure 1: The points used for discretizing the Dirichlet boundary condition.

In this paper we continue the approach started in [1] and [2], and develop a numerical method that directly discretizes the second order wave equation without first re-writing it as a system of first order equations. A Cartesian embedded boundary approach will be used to solve the above problem numerically. We cover the domain $\Omega$ by a regular Cartesian grid with points $\boldsymbol{x}_{i, j}=(i h, j h)^{T}$, where $i, j$ are integers and $h$ is the grid size. The boundary is allowed to intersect the grid in an arbitrary manner, see Figure 1. Centered finite differences are used to discretize the wave equation both in space and time and the boundary condition is approximated using straight forward interpolation. However, the details of how the boundary condition is discretized are important since they determine the accuracy and stability of the numerical method. In the current paper, we discretize the Dirichlet boundary condition such that both the solution and it's gradient become second order accurate. Furthermore, we add an artificial term to the discrete boundary condition that removes the stiffness due to small cells cut by the boundary, without sacrificing the second order accuracy of the method. As a result we can use an explicit time-integration method where the time-step essentially equals that of a periodic domain. Stability of the method is obtained by using a small fourth order stabilizing term of the form $h^{3} A^{T} A u_{t}$, where $A$ is the matrix representation of the discrete Laplace operator satisfying the discrete boundary condition [2].

The embedded boundary technique for discretizing partial differential equations date back to the first order technique by Weller and Shortley [3] and the higher order generalizations of Collatz [4]. More recently, several embedded boundary methods have been presented for various types of partial differential equations. For example, Pember et al. [5] used a Cartesian grid method for solving the time-dependent equations of gas dynamics. For the one and two-dimensional Euler equations, Berger, Helzel and LeVeque [6] developed a Cartesian "h-box" method which aims at avoiding the small-cell time step 
restriction without sacrificing accuracy. Zhang and LeVeque [7] solved the acoustic wave equation with discontinuous coefficients written as a first order system. A staggered grid method was used by Ditkowski, Dridi and Hesthaven [8] for solving Maxwell's equations on a Cartesian grid. The methods described in these papers all solve first order systems (in time). For Poisson's equation with Dirichlet boundary conditions, Johansen and Colella [9] derived an embedded boundary technique based on the finite volume method combined with multi-grid.

We have previously developed a Cartesian embedded boundary method for solving the wave equation subject to Dirichlet conditions [1]. That numerical method produces a solution that is second order accurate in max-norm, but the gradient of the solution is only first order accurate. Having accurate gradients is important in some application, such as Maxwell's equations, where the magnetic field depends on the curl of the electric field. There are two reasons why difference methods that use the embedded boundary discretization technique have problems to calculate gradients with full accuracy:

1. Even if the initial data are fully compatible with the boundary condition for the analytic problem, the spatial discretization can destroy the compatibility on the truncation error level, which can degrade the rate of convergence for the gradient. This problem occurs already in one space dimension and in Section 2 we will discuss how it can be avoided by using a "smooth start" procedure, where we change variables

$$
u(\boldsymbol{x}, t)=\tilde{u}(\boldsymbol{x}, t)+\left(u_{0}(\boldsymbol{x})+t u_{1}(\boldsymbol{x})\right) e^{-t^{2}},
$$

and numerically solve the problem for $\tilde{u}$. Since the new variable $\tilde{u}$ satisfies a modified wave equation subject to homogeneous initial conditions, there will be no incompatibilities on the truncation error level for the solution or it's first time derivative.

2. Because the boundary is embedded in a regular Cartesian grid, the truncation error in the boundary condition can oscillate wildly between consecutive grid points along the boundary. This problem occurs in two or more space dimensions and can again degrade the rate of convergence for the gradient. In Section 3, we first analyze this problem for Poisson's equation and then show that the wave equation behaves in the same way. The analysis shows that due to the highly oscillatory truncation error, the Dirichlet boundary condition must be discretized using a third order accurate formula to obtain a second order accurate gradient of the solution. The analysis also shows that a second order truncation error that varies smoothly along the boundary also results in a second order accurate gradient.

Guided by these results, we develop a new Cartesian embedded boundary method for the Dirichlet problem which we outline now. Let $v_{i, j}(t)$ denote the semi-discrete approximation of $u\left(x_{i}, y_{j}, t\right)$. A second order approximation of the Laplacian of $u$ is given by

$$
\Delta_{h} v_{i, j}=: \frac{1}{h^{2}}\left(v_{i+1, j}+v_{i-1, j}+v_{i, j+1}+v_{i, j-1}-4 v_{i, j}\right) .
$$


To be able to evaluate $\Delta_{h} v_{i, j}$ at all grid points inside $\Omega$, we use ghost points just outside the domain, see Figure 1. Let $x_{I}, x_{I I}$ be the intersections between the normal going through $\boldsymbol{x}_{i, j}$ and the grid lines $y=y_{j+1}$ and $y=y_{j+2}$, respectively. Denote by $\xi_{I}$, $\xi_{I I}=2 \xi_{I}$ the distances between $\boldsymbol{x}_{i, j}$ and $\left(x_{I}, y_{j+1}\right),\left(x_{I I}, y_{j+2}\right)$, respectively. To aid in the approximation of the Dirichlet condition, we construct a Lagrange interpolant between three points along the normal: $\left(0, v_{i, j}\right),\left(\xi_{I}, v_{I}\right),\left(\xi_{I I}, v_{I I}\right)$,

$$
L v=: g_{0}\left(\xi_{\Gamma}\right) v_{i, j}+g_{I}\left(\xi_{\Gamma}\right) v_{I}+g_{I I}\left(\xi_{\Gamma}\right) v_{I I},
$$

where the coefficients are given by

$$
g_{0}(\xi)=\frac{\left(\xi_{I}-\xi\right)\left(2 \xi_{I}-\xi\right)}{2 \xi_{I}^{2}}, \quad g_{I}(\xi)=\frac{\xi\left(2 \xi_{I}-\xi\right)}{\xi_{I}^{2}}, \quad g_{I I}(\xi)=\frac{\xi\left(\xi-\xi_{I}\right)}{2 \xi_{I}^{2}}
$$

To approximate $v_{I}$ and $v_{I I}$, we interpolate along the horizontal grid lines $y_{j+1}$ and $y_{j+2}$ :

$$
\begin{gathered}
v_{I}=c_{1} v_{i, j+1}+c_{2} v_{i+1, j+1}+c_{3} v_{i+2, j+1}, \\
v_{I I}=c_{4} v_{i, j+2}+c_{5} v_{i+1, j+2}+c_{6} v_{i+2, j+2} .
\end{gathered}
$$

where $c_{k}=\mathcal{O}(1)$ are the quadratic Lagrange interpolation coefficients. The interpolant is a third order approximation of the boundary value, i.e., $L u(t)=u\left(\boldsymbol{x}_{i, j}^{\Gamma}, t\right)+\mathcal{O}\left(h^{3}\right)$, where $\boldsymbol{x}_{i, j}^{\Gamma}$ is the intersection point between the boundary and the normal going through the ghost point $\boldsymbol{x}_{i, j}$.

The interpolation formulas for $v_{I}$ and $v_{I I}$, (9)-(10), hold when the angle $\theta$ between the normal and the $x$-axis satisfies $\pi / 4 \leq \theta \leq \pi / 2$. When $0 \leq \theta \leq \pi / 4$, the horizontal interpolations are replaced by corresponding interpolations in the vertical direction. The expressions in the remaining three quadrants are simply obtained by reflections in index space, leading to a total of 8 different cases to treat all possible directions of the boundary.

When the normal is close to vertical (or horizontal), the boundary can intersect the grid such that $\xi_{\Gamma}$ is arbitrarily close to $\xi_{I}$, i.e., $g_{0}\left(\xi_{\Gamma}\right)$ can be arbitrarily close to zero. Hence, if (7) would be used to approximate the boundary condition, the time-stepping of (1) would become very stiff. To mitigate the stiffness, we add an artificial term to the interpolant (7) and consider instead

$$
B_{h} v(t)=: g_{0}\left(\xi_{\Gamma}\right) v_{i, j}+g_{I}\left(\xi_{\Gamma}\right) v_{I}+g_{I I}\left(\xi_{\Gamma}\right) v_{I I}+\gamma\left(v_{i, j}-2 v_{I}+v_{I I}\right)=f\left(\boldsymbol{x}_{i, j}^{\Gamma}, t\right),
$$

where the constant $\gamma>0$. The artificial term is an undivided second difference in the normal direction so it inflicts an $\mathcal{O}\left(h^{2}\right)$ error in the boundary condition approximation. When the direction of the normal changes smoothly along the boundary, the error due to the artificial term also varies smoothly along the boundary. This is precisely the situation analyzed in Section 3, since the truncation error in the boundary condition is composed of a highly oscillatory $\mathcal{O}\left(h^{3}\right)$ part and a smooth $\mathcal{O}\left(h^{2}\right)$ component. The accuracy of both the solution and it's discrete gradient should therefore be $\mathcal{O}\left(h^{2}\right)$. 
Because of the artificial term in the boundary condition, the coefficient in front of the ghost point $v_{i, j}$ is bounded uniformly away from zero, since

$$
\gamma \leq g_{0}\left(\xi_{\Gamma}\right)+\gamma<1+\gamma
$$

As a result, the small cell stiffness problem is removed. By estimating the spectrum for the one-dimensional wave equation, we find that the eigenvalue with the largest magnitude is independent of small cells near the boundary when $\gamma \geq 0.25$, see Appendix A. Numerical computations indicate that $0.2 \leq \gamma \leq 0.5$ works well in practice, i.e., it is big enough to allow the time step to be chosen independently of the small cells near the boundary, but small enough to prevent the artificial term from dominating the error in the numerical solution.

We can use the boundary condition (11) to eliminate all ghost points in the discrete Laplacian (6), resulting in a semi-discrete approximation of (1)-(4),

$$
\begin{aligned}
\boldsymbol{v}_{t t} & =A \boldsymbol{v}+\boldsymbol{b}(t)+\boldsymbol{F}(t), & & t \geq 0 \\
\boldsymbol{v}(0) & =u_{0}(\boldsymbol{x}), & & \boldsymbol{x} \in \Omega, \\
\boldsymbol{v}_{t}(0) & =u_{1}(\boldsymbol{x}), & & \boldsymbol{x} \in \Omega .
\end{aligned}
$$

Here the vector $\boldsymbol{v}$ contains the solution at all grid points inside $\Omega, \boldsymbol{b}(t)$ is the discrete counterpart of the boundary forcing $f(\boldsymbol{x}, t)$ and $\boldsymbol{F}_{i, j}(t)=F\left(\boldsymbol{x}_{i, j}, t\right)$ for all $\boldsymbol{x}_{i, j} \in \Omega$.

We discretize the second order time derivative in (12) using a centered finite difference formula on the regular grid $t_{n}=n k, n=0,1,2, \ldots$, where $k>0$ is the time-step. Let $\boldsymbol{v}^{n}=\boldsymbol{v}\left(t_{n}\right)$. Because of the discretized form of the Dirichlet boundary condition, the matrix $A$ in (12) will not be symmetric. To avoid the resulting mild instability which was analyzed for the Neumann problem in [2], we use the discrete stabilization operator $h^{3} A^{T}\left(A\left(\boldsymbol{v}^{n}-\boldsymbol{v}^{n-1}\right) / k\right)$, which was proposed in the same paper. Note that this operator can be applied all the way up to the boundary. Away from the boundary, it is equivalent to $h^{3} \Delta_{h}^{2}\left(\boldsymbol{v}^{n}-\boldsymbol{v}^{n-1}\right) / k$, which is a very efficient damping term for highly oscillatory instabilities. For the general case with inhomogeneous boundary conditions and internal forcing, the proposed scheme becomes

$$
\frac{\boldsymbol{v}^{n+1}-2 \boldsymbol{v}^{n}+\boldsymbol{v}^{n-1}}{k^{2}}=A \boldsymbol{v}^{n}+\boldsymbol{b}\left(t_{n}\right)+\boldsymbol{F}\left(t_{n}\right)-\alpha h^{3} A^{T}\left(A\left(\boldsymbol{v}^{n}-\boldsymbol{v}^{n-1}\right) / k+\frac{d \boldsymbol{b}}{d t}\left(t_{n}\right)\right) \text {. }
$$

We note that the sparse structure of $A$ can be used to efficiently evaluate both $A \boldsymbol{v}$ and $A^{T} \boldsymbol{v}$, without the need to store the matrix explicitly, see [2]. Also note that this scheme bears many similarities to our method for the Neumann problem [2]. In particular, both methods use the same grid points for discretizing the boundary condition and the same type of stabilization term. Hence, the two methods can be combined in a straight forward manner to solve problems with mixed Neumann-Dirichlet conditions.

The remainder of the paper is organized as follows. The influence of incompatibilities between initial and boundary data on the truncation error level is analyzed in Section 2 
and the effect of highly oscillatory truncation errors in the boundary condition is studied in Section 3. Two-dimensional numerical experiments are performed in Section 4. We first construct a smooth solution against which the error in the numerical solution can be evaluated. The accuracy of the scheme and effects of the smooth start technique are evaluated in Section 4.1. In Section 4.2 we test the method by solving the $\mathrm{TM}_{z}$ problem for Maxwell's equations, i.e., the case when the electric field only has a component in the $z$-direction and the magnetic field has no component in the $z$-direction. The $\mathrm{TM}_{z}$ problem can be formulated as a scalar wave equation for the electric field in the $(x, y)$ plane, subject to a Dirichlet boundary condition. The wave equation for the electric field is coupled to ODE's for the magnetic field that are integrated in time as the electric field is evolved. This is a good test of the scheme, since the accuracy of the magnetic field is determined by the accuracy of the curl of the electric field. Numerical experiments are performed for a circular domain where the numerical solution is compared to an analytic solution, as well as for a more complex domain. Concluding remarks are presented in Section 5 .

\section{Smooth start}

We start by considering the one-dimensional half-plane problem

$$
u_{t t}=u_{x x}+F(x, t), \quad a \leq x<\infty, \quad t \geq 0,
$$

with initial data

$$
u(x, 0)=u_{0}(x), \quad u_{t}(x, 0)=u_{1}(x),
$$

and the boundary condition

$$
u(a, t)=f(t) .
$$

We assume that all data belongs to $C^{\infty}$. Then also the solution is smooth, provided compatibility conditions are satisfied. In the one-dimensional setting, these are

$$
\begin{aligned}
u(a, 0) & =u_{0}(a)=f(0), \\
u_{t}(a, 0) & =u_{1}(a)=f_{t}(0), \\
u_{t t}(a, 0) & =u_{0 x x}(a)+F(a, 0)=f_{t t}(0), \\
u_{t t t}(a, 0) & =u_{1 x x}(a)+F_{t}(a, 0)=f_{t t t}(0), \\
u_{t t t t}(a, 0) & =u_{0 x x x x}(a)+F_{x x}(a, 0)+F_{t t}(a, 0)=f_{t t t t}(0), \\
u_{t t t t t}(a, 0) & =u_{1 x x x x}(a)+F_{x x t}(a, 0)+F_{t t t}(a, 0)=f_{t t t t t}(0),
\end{aligned}
$$

We introduce a grid

$$
x_{\nu}=\nu h, \quad \nu=0,1,2, \ldots,
$$


where $h>0$ is the grid size. When the boundary is aligned with the grid, e.g. $a=0$, a second order centered finite difference approximation of (16)-(18) yields the expected second order convergence of the numerical solution and its gradient. However, we are interested in the case when the boundary is not aligned with the grid. We therefore take $a=(1-\alpha) h, 0<\alpha<1$, so that the boundary falls between $x_{0}$ and $x_{1}$. Hence, the grid point $x_{0}$ is outside the domain, but will be used as a ghost point to aid in the difference approximation. Let $v\left(x_{j}, t\right)$ be a grid function approximating $u\left(x_{j}, t\right)$. The one-dimensional counterpart of the boundary condition (11) is

$$
\begin{aligned}
B_{h}^{(1)} v(t)=: c_{0}(\alpha) v\left(x_{0}, t\right)+c_{1}(\alpha) & v\left(x_{1}, t\right)+c_{2}(\alpha) v\left(x_{2}, t\right) \\
& +\gamma\left(v\left(x_{0}, t\right)-2 v\left(x_{1}, t\right)+v\left(x_{2}, t\right)\right)=f(t), \quad \gamma>0
\end{aligned}
$$

where the coefficients satisfy

$$
c_{0}(\alpha)=\frac{1}{2} \alpha(1+\alpha), \quad c_{1}(\alpha)=(1-\alpha)(1+\alpha), \quad c_{2}(\alpha)=-\frac{1}{2} \alpha(1-\alpha) .
$$

Similar to the two-dimensional case, we remove the stiffness in the time-integration by taking $\gamma>0$ such that the coefficient in front of $v\left(x_{0}, t\right)$ is bounded away from zero for $0 \leq \alpha \leq 1$

The semi-discrete difference approximation of (16)-(18) when the boundary is not aligned with the grid becomes

$$
\begin{aligned}
v_{t t}\left(x_{\nu}, t\right) & =D_{+}^{x} D_{-}^{x} v\left(x_{\nu}, t\right)+F\left(x_{\nu}, t\right), \quad \nu=1,2, \ldots, \\
v\left(x_{\nu}, 0\right) & =u_{0}\left(x_{\nu}\right), \quad v_{t}\left(x_{\nu}, 0\right)=u_{1}\left(x_{\nu}\right), \quad \nu=1,2, \ldots, \\
B_{h}^{(1)} v(t) & =f(t),
\end{aligned}
$$

where $D_{+}^{x} v\left(x_{\nu}, t\right)=\left(v\left(x_{\nu+1}, t\right)-v\left(x_{\nu}, t\right)\right) / h$ is the usual forward divided difference operator in the $x$-direction, and $D_{-}^{x} v\left(x_{\nu}, t\right)=D_{+}^{x} v\left(x_{\nu-1}, t\right)$. For later use, we also define the centered operator $D_{0}^{x} v\left(x_{\nu}, t\right)=\left(v\left(x_{\nu+1}, t\right)-v\left(x_{\nu-1}, t\right) /(2 h)\right.$. Using Taylor series expansions, we see that the error $e\left(x_{\nu}, t\right)=u\left(x_{\nu}, t\right)-v\left(x_{\nu}, t\right)$ satisfies

$$
\begin{aligned}
e_{t t}\left(x_{\nu}, t\right) & =D_{+}^{x} D_{-}^{x} e\left(x_{\nu}, t\right)-\frac{h^{2}}{12} u_{x x x x}\left(x_{\nu}, t\right)+\mathcal{O}\left(h^{4}\right), \quad \nu=1,2, \ldots, \\
e\left(x_{\nu}, 0\right) & =e_{t}\left(x_{\nu}, 0\right)=0, \quad \nu=1,2, \ldots \\
B_{h}^{(1)} e(t) & =\gamma h^{2} u_{x x}(a, t)+h^{3}(C+\gamma \alpha) u_{x x x}(a, t)+\mathcal{O}\left(h^{4}\right) .
\end{aligned}
$$

Since the data is $\mathcal{O}\left(h^{2}\right),|e(\cdot, t)|_{\infty}=\mathcal{O}\left(h^{2}\right)$. To derive an estimate for the gradient of $e$, we first derive an equation for $w=e_{t t}$. By taking two time-derivatives of the differential equation (26) and the boundary condition (28), we get

$$
\begin{aligned}
w_{t t}\left(x_{\nu}, t\right) & =D_{+}^{x} D_{-}^{x} w\left(x_{\nu}, t\right)-\frac{h^{2}}{12} u_{x x x x t t}\left(x_{\nu}, t\right)+\mathcal{O}\left(h^{4}\right), \quad \nu=1,2, \ldots, \\
B_{h}^{(1)} w(t) & =\gamma h^{2} u_{x x t t}(a, t)+h^{3}(C+\gamma \alpha) u_{x x x t t}(a, t)+\mathcal{O}\left(h^{4}\right) .
\end{aligned}
$$


To derive initial data for $w$, we can apply the differential equation (26) to the initial data (27). However, this only applies away from the boundary. For $\nu \geq 2$ we have

$$
\begin{aligned}
w\left(x_{\nu}, 0\right) & =-\frac{h^{2}}{12} u_{x x x x}\left(x_{\nu}, 0\right)+\mathcal{O}\left(h^{4}\right), \quad \nu=2,3, \ldots \\
w_{t}\left(x_{\nu}, 0\right) & =-\frac{h^{2}}{12} u_{x x x x t}\left(x_{\nu}, 0\right)+\mathcal{O}\left(h^{4}\right), \quad \nu=2,3, \ldots
\end{aligned}
$$

To evaluate the initial data at the first interior point $x_{1}$, we must first apply the boundary condition (28) to define $e\left(x_{0}, 0\right)$,

$$
\begin{aligned}
B_{h}^{(1)} e(0)=:\left(c_{0}+\gamma\right) e\left(x_{0}, 0\right)+\left(c_{1}-2 \gamma\right) e & \left(x_{1}, 0\right)+\left(c_{2}+\gamma\right) e\left(x_{2}, 0\right)= \\
& \gamma h^{2} u_{x x}(a, 0)+h^{3}(C+\gamma \alpha) u_{x x x}(a, 0)+\mathcal{O}\left(h^{4}\right)
\end{aligned}
$$

Since $e\left(x_{1}, 0\right)=e\left(x_{2}, 0\right)=0, D_{+}^{x} D_{-}^{x} e\left(x_{1}, 0\right)=e\left(x_{0}, 0\right) / h^{2}$. Therefore, (26) gives us

$$
w\left(x_{1}, 0\right)=e_{t t}\left(x_{1}, 0\right)=\frac{\gamma}{c_{0}+\gamma} u_{x x}(a, 0)+\frac{h(C+\gamma \alpha)}{c_{0}+\gamma} u_{x x x}(a, 0)+\mathcal{O}\left(h^{2}\right) .
$$

By taking the time derivative of the boundary condition, the same procedure can be used to obtain

$$
w_{t}\left(x_{1}, 0\right)=e_{t t t}\left(x_{1}, 0\right)=\frac{\gamma}{c_{0}+\gamma} u_{x x t}(a, 0)+\frac{h(C+\gamma \alpha)}{c_{0}+\gamma} u_{x x x t}(a, 0)+\mathcal{O}\left(h^{2}\right) .
$$

Hence, in general the forcing in the initial data is $\mathcal{O}(1)$ at the first interior point, which makes $|w(\cdot, t)|_{\infty}=\mathcal{O}(1)$. Consequently, $\left|D_{+}^{x} D_{-}^{x} e(\cdot, t)\right|_{\infty}=\mathcal{O}(1)$ and we can not obtain the desired estimate for the gradient of $e$.

The situation is much better in the case with homogeneous initial data, $u_{0}(x)=$ $u_{1}(x)=0$. Now all spatial derivatives of $u$ and $u_{t}$ are initially zero, so the initial data for $w$ are

$$
w\left(x_{\nu}, 0\right)=w_{t}\left(x_{\nu}, 0\right)=0, \quad \nu=1,2, \ldots
$$

Hence the forcing for $w$ is $\mathcal{O}\left(h^{2}\right)$. Therefore we can derive an estimate for $\left|e_{t t}(\cdot, t)\right|_{\infty}=$ $|w(\cdot, t)|_{\infty}=\mathcal{O}\left(h^{2}\right)$ and

$$
\left|D_{+}^{x} D_{-}^{x} e(\cdot, t)\right|_{\infty} \leq|w(\cdot, t)|_{\infty}+\frac{h^{2}}{12}\left|u_{x x x x}(\cdot, t)\right|_{\infty}=\mathcal{O}\left(h^{2}\right) .
$$

Since $\left|D_{+}^{x} e(\cdot, t)\right|_{\infty}$ can be estimated in terms of $e$ and $D_{+}^{x} D_{-}^{x} e$, we have

$$
\left|D_{+}^{x} e\right|_{\infty}=\mathcal{O}\left(h^{2}\right)
$$

A problem with general, but compatible, initial data can be reformulated into a problem with homogeneous initial data by changing variables according to (5). If the original 
variable $u$ satisfies (1)-(4), the variable $\tilde{u}$ satisfies the modified problem

$$
\begin{array}{rlrlrl}
\tilde{u}_{t t} & =\Delta \tilde{u}+\tilde{F}(\boldsymbol{x}, t), & & \boldsymbol{x} \in \Omega, & t \geq 0 \\
\tilde{u}(\boldsymbol{x}, t) & =\tilde{f}(\boldsymbol{x}, t), & & \boldsymbol{x} \in \Gamma, \quad t \geq 0, \\
\tilde{u}(\boldsymbol{x}, 0) & =0, & & \boldsymbol{x} \in \Omega, & \\
\tilde{u}_{t}(\boldsymbol{x}, 0) & =0, & & \boldsymbol{x} \in \Omega, &
\end{array}
$$

where the modified internal forcing function is

$$
\tilde{F}(\boldsymbol{x}, t)=F(\boldsymbol{x}, t)+\left[\Delta u_{0}(\boldsymbol{x})+t \Delta u_{1}(\boldsymbol{x})+\left(2-4 t^{2}\right) u_{0}(\boldsymbol{x})+\left(6 t-4 t^{3}\right) u_{1}(\boldsymbol{x})\right] e^{-t^{2}},
$$

and the boundary forcing becomes

$$
\tilde{f}(\boldsymbol{x}, t)=f(\boldsymbol{x}, t)-\left[u_{0}(\boldsymbol{x})+t u_{1}(\boldsymbol{x})\right] e^{-t^{2}}, \quad \boldsymbol{x} \in \Gamma .
$$

To solve the problem for $u(\boldsymbol{x}, t)$, we first compute $\tilde{u}(\boldsymbol{x}, t)$ numerically and then add in $\left(u_{0}(\boldsymbol{x})+t u_{1}(\boldsymbol{x})\right) e^{-t^{2}}$ to obtain the solution of the original problem. By doing so, we obtain a second order accurate solution where also the gradient and second derivative of the numerical solution are second order accurate.

To verify the above theory, we solve the embedded boundary problem (23)-(25) numerically. Now, we consider the bounded domain

$$
a \leq x \leq b
$$

and add a boundary condition at $x=b$,

$$
u(b, t)=f_{2}(t) .
$$

The interior grid points are $q_{0} \leq \nu \leq q_{1}$ such that $a+\alpha h=x_{q_{0}}$ and $x_{q_{1}}+\beta h=b$, $0<\beta<1$. We take initial data, interior and boundary forcing functions such that the analytic problem is solved by $u(x, t)=\sin (2 x-t+\pi / 4)$, i.e.,

$$
\begin{aligned}
F\left(x_{\nu}, t\right) & =3 \sin \left(2 x_{\nu}-t+\pi / 4\right), \\
f(t) & =\sin (2 a-t+\pi / 4), \\
f_{2}(t) & =\sin (2 b-t+\pi / 4), \\
u_{0}\left(x_{\nu}\right) & =\sin \left(2 x_{\nu}+\pi / 4\right), \quad u_{1}\left(x_{\nu}\right)=-\cos \left(2 x_{\nu}+\pi / 4\right) .
\end{aligned}
$$

We introduce a grid in time $t_{n}=n k, k>0$ and discretize time-derivatives by centered differences. We set $h=2 \pi / N$, where $N$ is a positive integer, so that $x_{N}=2 \pi$. The boundary condition at $x=a$ is discretized by (21), shifted to involve the points $\left(q_{0}-\right.$ $\left.1, q_{0}, q_{0}+1\right)$. The boundary condition at $x=b$ is discretized using a corresponding formula 


\begin{tabular}{c|c|c|c|c|c}
$N$ & $\|u-v\|_{\infty}$ & $\left\|\frac{\partial u}{\partial x}-D_{0}^{x} v\right\|_{\infty}$ & $\left\|\frac{\partial^{2} u}{\partial x^{2}}-D_{+}^{x} D_{-}^{x} v\right\|_{\infty}$ & $\alpha$ & $\beta$ \\
\hline 200 & $1.22 \times 10^{-3}$ & $9.36 \times 10^{-3}$ & $1.88 \times 10^{0}$ & 0.222 & 0.338 \\
400 & $2.53 \times 10^{-4}$ & $3.13 \times 10^{-3}$ & $3.59 \times 10^{-1}$ & 0.445 & 0.676 \\
800 & $6.29 \times 10^{-5}$ & $1.26 \times 10^{-3}$ & $2.98 \times 10^{-1}$ & 0.890 & 0.352 \\
\hline
\end{tabular}

Table 1: Direct start: Error in the computed solution when the boundary is embedded between grid points. The errors are reported at time $t=6.333$, the domain is $0.37 \leq x \leq$ $5.1, \gamma=0.2$, and $k / h=0.9$.

\begin{tabular}{c|c|c|c|c|c}
$N$ & $\|u-v\|_{\infty}$ & $\left\|\frac{\partial u}{\partial x}-D_{0}^{x} v\right\|_{\infty}$ & $\left\|\frac{\partial^{2} u}{\partial x^{2}}-D_{+}^{x} D_{-}^{x} v\right\|_{\infty}$ & $\alpha$ & $\beta$ \\
\hline 200 & $8.90 \times 10^{-4}$ & $1.14 \times 10^{-3}$ & $1.02 \times 10^{-3}$ & 0.222 & 0.338 \\
400 & $2.22 \times 10^{-4}$ & $2.86 \times 10^{-4}$ & $2.56 \times 10^{-4}$ & 0.445 & 0.676 \\
800 & $5.55 \times 10^{-5}$ & $7.09 \times 10^{-5}$ & $6.38 \times 10^{-5}$ & 0.890 & 0.352 \\
\hline
\end{tabular}

Table 2: Smooth start: Error in the computed solution when the boundary is embedded between grid points. The errors are reported at time $t=6.333$, the domain is $0.37 \leq x \leq$ $5.1, \gamma=0.2$, and $k / h=0.9$.

involving the points $\left(q_{1}-1, q_{1}, q_{1}+1\right)$ with $\beta$ replacing $\alpha$. We arrive at the explicit time integration scheme

$$
\begin{aligned}
& D_{+}^{t} D_{-}^{t} v\left(x_{\nu}, t_{n}\right)=D_{+}^{x} D_{-}^{x} v\left(x_{\nu}, t_{n}\right)+F\left(x_{\nu}, t_{n}\right), \quad \nu=q_{0}, q_{0}+1, \ldots, q_{1} \\
& v\left(x_{\nu}, 0\right)=u_{0}\left(x_{\nu}\right), \quad \nu=q_{0}, q_{0}+1, \ldots, q_{1} \\
& v\left(x_{\nu},-k\right)= u_{0}\left(x_{\nu}\right)-k u_{1}\left(x_{\nu}\right)+\frac{k^{2}}{2}\left(u_{0 x x}\left(x_{\nu}, 0\right)+F\left(x_{\nu}, 0\right)\right)- \\
& \frac{k^{3}}{6}\left(u_{1 x x}\left(x_{\nu}, 0\right)+F_{t}\left(x_{\nu}, 0\right)\right), \quad \nu=q_{0}, q_{0}+1, \ldots, q_{1} \\
& B_{h}^{(a)} v\left(t_{n}\right)= f\left(t_{n}\right), \quad B_{h}^{(b)} v\left(t_{n}\right)=f_{2}\left(t_{n}\right) .
\end{aligned}
$$

One can show that the above scheme is stable for $k / h<1$, cf. [1]. Numerical computations, see Table 1, indicate that the solution is second order accurate, but the gradient is only a little better than first order accurate and the second derivative has an $\mathcal{O}(1)$ error. However, by using the smooth start procedure, i.e., change variables according to (5) and numerically solving (29)-(32), second order accuracy is also obtained for the gradient and the second derivative, see Table 2.

We have also observed that the convergence of the gradient improves when the initial data is inhomogeneous, but the interior and boundary forcing functions are homogeneous, $F(x, t)=0, f(t)=0$. From the compatibility relations (19), we see that all even spatial 
derivatives of $u$ and $u_{t}$ are zero at $x=a, t=0$. In particular, $u_{x x}(a, 0)=0$ and $u_{x x t}(a, 0)=0$, so the leading order terms in the initial data for $w$ become one order smaller in $h$. Hence, $w\left(x_{1}, 0\right)=\mathcal{O}(h)$ and $w_{t}\left(x_{1}, 0\right)=\mathcal{O}(h)$, and we get $|w(\cdot, t)|_{\infty}=\mathcal{O}(h)$. Hence, $|e(\cdot, t)|_{\infty}=\mathcal{O}\left(h^{2}\right)$ but $\left|D_{+}^{x} D_{-}^{x} e(\cdot, t)\right|_{\infty}=\mathcal{O}(h)$. Numerical experiments suggest that the solution and its gradient are second order accurate but the second derivative is only first order accurate. Naturally, the error in the second derivative can be made second order accurate also in this case by using smooth start.

There are also cases when the smooth start procedure is not necessary. This occurs, for example, when the boundary data is homogeneous, $f(t)=0$, and the internal forcing and initial data have compact support in the interior of $\Omega$.

\section{An analytic model of the Dirichlet problem}

In this section we first consider Poisson's equation

$$
u_{x x}+u_{y y}=F(x, y),
$$

on the half-plane $y-2 x \leq 0$ with Dirichlet boundary conditions

$$
u(x, y)=f(x, y) \quad \text { for } \quad y=2 x .
$$

We want to solve the problem numerically, use the same Cartesian grid as before, and approximate (33) by the second order approximation

$$
\Delta_{h} v\left(x_{m}, y_{l}\right)=F_{h}\left(x_{m}, y_{l}\right)
$$

in all interior points. Here $F_{h}$ is the restriction of $F$ to the grid.

The grid is not aligned with the boundary. Along the boundary only every second point is on the grid. A second order accurate approximation of (34) is, for example, given by

$$
\begin{aligned}
v(l h, 2 l h) & =f(l h, 2 l h), \\
\frac{1}{2}\{v((l-1) h,(2 l-1) h)+v(l h,(2 l-1) h)\} & =f\left(\left(l-\frac{1}{2}\right) h,(2 l-1) h\right),
\end{aligned}
$$

for $l=0, \pm 1, \pm 2, \ldots$. We write (35)-(36) formally as

$$
B_{h} v=f_{h} .
$$

To obtain an error estimate we assume that the solution of (33)-(34) is smooth. (This is, for example, the case if $F, f$ are smooth and decay rapidly as $|x|+|y| \rightarrow \infty$, cf. [10].) The usual truncation error analysis gives us, for the error $e=v-u$ on the grid,

$$
\begin{aligned}
& \Delta_{h} e=h^{2} G \quad \text { in the interior, } \\
& B_{h} e=h^{2} g \quad \text { on the boundary. }
\end{aligned}
$$


Here $G$ is the restriction of a smooth function to the grid but $g$ is in general not smooth. The boundary condition (36) implies that $g$ oscillates between 0 and $u_{x x}+\mathcal{O}\left(h^{2}\right)$ from grid point to grid point. This rapid change is typical for embedded boundary difference approximations in general domains.

If we only want to estimate $e$, this is tolerable because one can prove, using the discrete maximum principle, that the error is $\mathcal{O}\left(h^{2}\right)$. However, if we also want to calculate the gradient, then the error degrades, in general, to $\mathcal{O}(h)$. This degradation does not depend on $G$. If $G \neq 0$, we solve an auxiliary problem

$$
\begin{aligned}
& \Delta_{h} e_{1}=h^{2} G, \quad \text { in the interior, } \\
& B_{h} e_{1}=0, \quad \text { on the boundary. }
\end{aligned}
$$

The problem (39)-(40) is a second order accurate approximation of

$$
\begin{aligned}
\Delta u_{1} & =h^{2} G, \quad \text { in the interior, } \\
u_{1} & =0, \quad \text { on the boundary. }
\end{aligned}
$$

The function $u_{1}$ and its gradient are of order $\mathcal{O}\left(h^{2}\right)$. Since $e_{1}-u_{1}=\mathcal{O}\left(h^{4}\right)$, on the grid, the discrete gradient of $e_{1}$ is $\mathcal{O}\left(h^{2}\right)$. Subtracting the solution of (39)-(40) from the solution of (37)-(38) shows that we can assume that $G \equiv 0$, i.e., we discuss

$$
\begin{aligned}
& \Delta_{h} e=0, \quad \text { in the interior, } \\
& B_{h} e=h^{2} g, \quad \text { on the boundary. }
\end{aligned}
$$

To gain insight into the effects of highly oscillatory boundary data, we analyze a modified equation model of (41)-(42). A more sophisticated analysis, where the discrete nature of the problem is taken into account, will be presented elsewhere. The problem (41)-(42) is a second order accurate approximation of

$$
\begin{gathered}
\Delta e=0, \quad y-2 x \leq 0, \\
e=h^{2} g, \quad y=2 x,
\end{gathered}
$$

and we proceed by studying the properties of its solution, in particular when $g$ is highly oscillatory. To simplify the notation, we rotate the coordinate system and introduce new independent variables

$$
\begin{aligned}
& \xi=(2 x-y) / \sqrt{5}, \\
& \eta=(x+2 y) / \sqrt{5} .
\end{aligned}
$$

The boundary is aligned with $\xi=0$ and we consider the half-plane problem for $\xi \geq 0$. We assume that all functions are $2 \pi$-periodic in $\eta$. Fourier transforming (43)-(44) in $\eta$ gives us

$$
\begin{aligned}
\hat{e}_{\xi \xi}-\omega^{2} \hat{e} & =0, \quad \omega \text { integer }, \\
\hat{e}(0, \omega) & =h^{2} \hat{g}(\omega) .
\end{aligned}
$$


Since we are mainly interested in highly oscillatory $g$, we always assume that $\hat{g}(0)=0$. Then the unique solution in $L_{2}$ is given by

$$
e=h^{2} \sum_{\omega \neq 0} e^{-|\omega| \xi+i \omega \eta} \hat{g}(\omega)
$$

and we have

$$
e_{\xi}=-h^{2} \sum_{\omega \neq 0}|\omega| e^{-|\omega| \xi+i \omega \eta} \hat{g}(\omega)
$$

We make the following observations:

1) If the boundary data are smooth, i.e., $\hat{g}(\omega)$ decays rapidly, then the solution is also smooth up to the boundary.

2) If we only know that $g \in L_{2}$, then we obtain, for every fixed $\xi$,

$$
\begin{aligned}
\left\|e_{\xi}(\xi, \cdot)\right\|^{2} & =\int_{-\pi}^{\pi}\left|e_{\xi}(\xi, \eta)\right|^{2} d \eta=\sum_{\omega \neq 0}|\omega|^{2} e^{-2|\omega| \xi}\left|h^{2} \hat{g}(\omega)\right|^{2} \\
& =\frac{1}{\xi^{2}} \sum_{\omega \neq 0}|\xi \omega|^{2} e^{-2|\omega| \xi}\left|h^{2} \hat{g}(\omega)\right|^{2} \leq \frac{\text { const. }}{\xi^{2}} \sum_{\omega \neq 0} e^{-|\omega| \xi}\left|h^{2} \hat{g}(\omega)\right|^{2} .
\end{aligned}
$$

Thus the solution becomes smooth away from the boundary layer at $\xi=0$.

3) If the boundary data are highly oscillatory, i.e.,

$$
g(\eta)=\sum_{|\omega| \geq 1 / h} e^{i \omega \eta} \hat{g}(\omega), \quad 0<h<<1
$$

we obtain

$$
\begin{aligned}
\left\|e_{\xi}(\xi, \cdot)\right\|^{2} & \leq \frac{\text { const. }}{\xi^{2}} \sum_{|\omega| \geq 1 / h} e^{-|\omega| \xi}\left|h^{2} \hat{g}(\omega)\right|^{2} \\
& \leq \frac{\text { const. }}{\xi^{2}} e^{-\xi / h} \sum_{|\omega| \geq 1 / h} e^{-(|\omega|-1 / h) \xi}\left|h^{2} \hat{g}(\omega)\right|^{2}
\end{aligned}
$$

i.e., the thickness of the boundary layer is $\mathcal{O}(h)$.

4) The highest frequency on a grid is $|\omega h|=\pi$. Hence, if $\hat{g}(\omega)=0$ for $|\omega|>\pi / h$, then

$$
\left\|e_{\xi}(\xi, \cdot)\right\|^{2}=\frac{1}{h^{2}} \sum_{|\omega h| \leq \pi}|\omega h|^{2} e^{-2|\omega| \xi}\left|h^{2} \hat{g}(\omega)\right|^{2} \leq \pi^{2} \sum_{|\omega h| \leq \pi} e^{-2|\omega| \xi}|h \hat{g}(\omega)|^{2} .
$$

Thus the gradient is of the order $\mathcal{O}(h)$. 
To summarize our results for the Dirichlet problem, the gradient is only of the order $\mathcal{O}\left(h^{2}\right)$ if the boundary data $h^{2} g(\eta)$ is the restriction of a smooth function. For highly oscillatory $g(\eta)$, the gradient will be $\mathcal{O}(h)$. We can also use a third order accurate approximation of the boundary condition. Then the solution is of the order $\mathcal{O}\left(h^{3}\right)$ and the discrete gradient is of the order $\mathcal{O}\left(h^{2}\right)$, even if the boundary data is highly oscillatory. Since the problem is linear, we also get a discrete gradient of the order $\mathcal{O}\left(h^{2}\right)$ when the boundary data consists of a smooth $\mathcal{O}\left(h^{2}\right)$ term together with a highly oscillatory $\mathcal{O}\left(h^{3}\right)$ component.

\subsection{The wave equation}

We next consider the wave equation (1)-(4) on the half-plane $x \geq 0,-\infty<y<\infty$. In this case, the Dirichlet boundary condition is

$$
u(0, y, t)=f(y, t)
$$

We assume that all data are $2 \pi$-periodic functions in $y$, have compact support, and that they can be extended smoothly to the whole plane. Therefore we can assume that

$$
F \equiv u_{0} \equiv u_{1} \equiv 0
$$

Otherwise we would first solve the Cauchy problem which we would then subtract from $u$. Thus we consider

$$
\begin{aligned}
u_{t t} & =\Delta u, \\
u(x, y, 0) & =u_{t}(x, y, 0)=0, \\
u(0, y, t) & =f(y, t) .
\end{aligned}
$$

If $f(y, 0) \neq 0$, then the boundary data are not compatible with the initial data. If we want the solution to have $p$ bounded time derivatives, we need that

$$
\partial^{j} f(y, t) /\left.\partial t^{j}\right|_{t=0}=0, \quad j=0,1,2, \ldots, p-1 .
$$

We Fourier transform the problem in the $y$-direction and obtain

$$
\begin{aligned}
\hat{u}_{t t} & =\hat{u}_{x x}-\omega^{2} \hat{u}, \\
\hat{u}(x, \omega, 0) & =\hat{u}_{t}(x, \omega, 0)=0, \\
\hat{u}(0, \omega, t) & =\hat{f}(\omega, t),
\end{aligned}
$$

with

$$
\partial^{j} \hat{f}(\omega, t) /\left.\partial t^{j}\right|_{t=0}=0, \quad j=0,1,2, \ldots, p-1 .
$$

Because of the compatibility assumption (49), the solution will be smooth in time. If the boundary data is smooth in $y$, i.e., the Fourier coefficients $\hat{f}(\omega, t)$ decay rapidly 
for large $\omega$, the solution will also be smooth in space. However, as we have seen before, the truncation error in the boundary condition is highly oscillatory, and we proceed by studying large $|\omega|$.

When $|\omega| \gg 1$, we solve (50) by iteration. For $j=1,2, \ldots$, let

$$
\begin{aligned}
\hat{u}_{x x}^{(j)}-\omega^{2} \hat{u}^{(j)} & =\hat{u}_{t t}^{(j-1)}, \\
\hat{u}^{(j)}(0, \omega, t) & =\hat{f}(\omega, t) .
\end{aligned}
$$

We take $\hat{u}^{(0)}=0$, so $\hat{u}^{(1)}$ satisfies

$$
\begin{aligned}
\hat{u}_{x x}^{(1)}-\omega^{2} \hat{u}^{(1)} & =0, \\
\hat{u}^{(1)}(0, \omega, t) & =\hat{f}(\omega, t) .
\end{aligned}
$$

The only bounded solution is $\hat{u}^{(1)}(x, \omega, t)=\hat{f}(\omega, t) e^{-|\omega| x}$. The problem for $\hat{u}^{(2)}$ becomes

$$
\begin{aligned}
\hat{u}_{x x}^{(2)}-\omega^{2} \hat{u}^{(2)} & =\hat{f}_{t t}(\omega, t) e^{-|\omega| x}, \\
\hat{u}^{(2)}(0, \omega, t) & =\hat{f}(\omega, t),
\end{aligned}
$$

which is solved by

$$
\hat{u}^{(2)}=\hat{u}^{(1)}-\frac{1}{2 \omega^{2}} \hat{f}_{t t}|\omega| x e^{-|\omega| x} .
$$

Note that $\alpha e^{-\alpha} \leq$ const. for $\alpha \geq 0$. Hence, for $|\omega| \gg 1$, the solution of (50) is

$$
\hat{u}(x, \omega, t)=\hat{f} e^{-|\omega| x}-\frac{1}{2 \omega^{2}} \hat{f}_{t t}|\omega| x e^{-|\omega| x}+\mathcal{O}\left(\frac{1}{\omega^{4}}\right),
$$

with gradient

$$
\hat{u}_{x}(x, \omega, t)=-|\omega| \hat{f} e^{-|\omega| x}+\mathcal{O}\left(\frac{1}{|\omega|}\right) .
$$

Therefore, the properties of the gradient of $\hat{u}$ can be reduced to the properties of $\hat{u}^{(1)}$, which was studied in the previous section. An important consequence of this is that the gradient of $u$ only becomes large in a boundary layer near $x=0$, while the solution remains smooth in the interior of the domain.

\section{Numerical experiments}

In this section we numerically solve (1)-(4) with the scheme described above. We denote the CFL-number by $\mathrm{CFL} \equiv k / h$. Note that for a two-dimensional periodic domain our time-integration scheme $(15)$ is stable for $\mathrm{CFL} \leq 1 / \sqrt{2} \approx 0.71$. Also note that all errors reported below are measured in max-norm over all grid points inside $\Omega$. 
We start the time-integration at $n=0$ and take $v_{i, j}^{0}=u_{0}\left(x_{i}, y_{j}\right)$. We need to use a fourth order accurate approximation of $u\left(x_{i}, y_{j},-k\right)$ for $v_{i, j}^{-1}$. This is achieved by using the differential equation to approximate the second and third time derivatives,

$$
\begin{aligned}
v_{i, j}^{-1}=u_{0}\left(x_{i}, y_{j}\right)-k u_{1}\left(x_{i}, y_{j}\right)+ & \frac{k^{2}}{2}\left(D_{+}^{x} D_{-}^{x}+D_{+}^{y} D_{-}^{y}\right) u_{0}\left(x_{i}, y_{j}\right)+\frac{k^{2}}{2} F\left(\boldsymbol{x}_{i, j}, 0\right) \\
& -\frac{k^{3}}{6}\left(\left(D_{+}^{x} D_{-}^{x}+D_{+}^{y} D_{-}^{y}\right) u_{1}\left(x_{i}, y_{j}\right)-\frac{k^{3}}{6} F_{t}\left(\boldsymbol{x}_{i, j}, 0\right) .\right.
\end{aligned}
$$

Note that when the smooth start procedure is employed, the initial data is homogeneous, so the above formula simplifies to

$$
\tilde{v}_{i, j}^{-1}=\frac{k^{2}}{2} \tilde{F}\left(\boldsymbol{x}_{i, j}, 0\right)-\frac{k^{3}}{6} \tilde{F}_{t}\left(\boldsymbol{x}_{i, j}, 0\right),
$$

where the modified forcing initially satisfies

$$
\tilde{F}(\boldsymbol{x}, 0)=F(\boldsymbol{x}, 0)+2 u_{0}(\boldsymbol{x})+\Delta u_{0}(\boldsymbol{x}), \quad \tilde{F}_{t}(\boldsymbol{x}, 0)=F_{t}(\boldsymbol{x}, 0)+6 u_{1}(\boldsymbol{x})+\Delta u_{1}(\boldsymbol{x}) .
$$

To use the smooth start procedure, we need to evaluate $\Delta u_{k}, k=0,1$, at all interior grid points. When $u_{0}(x, y)$ and $u_{1}(x, y)$ are complicated analytic expressions of $x$ and $y$, an appealing alternative is to approximate $\Delta u_{k}$ by finite differences. Numerical experiments indicate that a centered second or forth order accurate difference approximation of $\Delta u_{k}$, $k=0,1$, can replace analytic expressions without any noticeable degradation of the computed solutions, as long as $u_{0}$ and $u_{1}$ are well resolved on the grid. However, to define all values in the five or seven-point formula for the centered difference approximation of the Laplacian, this approach only works if $u_{0}$ and $u_{1}$ can be evaluated at all interior grid points as well as one or two grid points outside the boundary, respectively.

\subsection{Trigonometric exact solution}

To evaluate the accuracy of the method and the properties of the smooth start technique, we begin by considering the case when the exact solution is known. Given a smooth function $U(\boldsymbol{x}, t)$, this is accomplished by constructing balancing interior and boundary forcing functions $F(\boldsymbol{x}, t)=U_{t t}(\boldsymbol{x}, t)-\Delta U(\boldsymbol{x}, t)$ and $f\left(\boldsymbol{x}_{\Gamma}, t\right)=U\left(\boldsymbol{x}_{\Gamma}, t\right)$, respectively. The initial data are obtained by setting $u_{0}(\boldsymbol{x})=U(\boldsymbol{x}, 0), u_{1}(\boldsymbol{x})=U_{t}(\boldsymbol{x}, 0)$. The continuous problem is then discretized and the error in the discrete solution can be obtained by taking the difference between the numerical and analytic solutions at each grid point.

We choose the exact solution to be the trigonometric traveling wave

$$
U(x, y, t)=\sin (\omega(x-t)) \sin (\omega y), \quad \omega=2 \pi .
$$

The domain $\Omega$ is taken to be an ellipse centered at the origin with semi-axes $x_{s}=1$ and $y_{s}=0.75$. The Cartesian grid covers the rectangle $-1.04 \leq x \leq 1.04,-0.78 \leq y \leq 0.78$, 


\begin{tabular}{c|c|c|c||c|c|c}
\multicolumn{5}{c||}{ Direct start } & \multicolumn{3}{c}{ Smooth start } \\
\hline$N$ & $\left\|u_{\mathrm{err}}\right\|_{\infty}$ & $\left\|\nabla u_{\mathrm{err}}\right\|_{\infty}$ & $\left\|\Delta u_{\mathrm{err}}\right\|_{\infty}$ & $\left\|u_{\mathrm{err}}\right\|_{\infty}$ & $\left\|\nabla u_{\mathrm{err}}\right\|_{\infty}$ & $\left\|\Delta u_{\mathrm{err}}\right\|_{\infty}$ \\
\hline 201 & $2.23 \times 10^{-3}$ & $3.48 \times 10^{-2}$ & 14.3 & $1.39 \times 10^{-3}$ & $8.60 \times 10^{-3}$ & $7.25 \times 10^{-2}$ \\
401 & $5.38 \times 10^{-4}$ & $1.27 \times 10^{-2}$ & 9.86 & $3.43 \times 10^{-4}$ & $2.15 \times 10^{-3}$ & $1.83 \times 10^{-2}$ \\
\hline
\end{tabular}

Table 3: Grid refinement study showing the errors in the computed solutions at $\mathrm{t}=2.0$ when the exact solution is the trigonometric function (52), with direct and smooth start.

the grid size is $h=2.08 /(N-1)$ and $\mathrm{CFL}=0.5$. To asses the accuracy of the scheme we run the computations until time $t=2.0$. Note that no damping is necessary for these short runs $(\alpha=0)$. In Table 3, we present a grid refinement study for the scheme (15) started directly (from inhomogeneous initial data) and smoothly, respectively. In both cases, the solution itself converges as $\mathcal{O}\left(h^{2}\right)$, but the gradient and Laplacian are only second order accurate when smooth start is being used. (The second order formulas $D_{0}^{x} v_{i, j}$ and $D_{0}^{y} v_{i, j}$ are used to approximate the discrete gradient, and the component of the gradient with the largest error is reported. The Laplacian is approximated by $\Delta_{h} v_{i, j}$.) Note that when the computation is started directly, the error in the gradient is only a little better than first order accurate and the error in the Laplacian is $\mathcal{O}(1)$. This agrees with our observations for the one-dimensional case, cf. Table 1.

To more clearly see the impact of the smooth start procedure on the solution, in Figure 2 we plot the error in the solution at time $t=2.0$, with direct and smooth start. Clearly, the smooth start procedure efficiently removes the high frequency errors present in the computation using direct start.

Next we investigate the long-time properties of the time integration scheme. In Figure 3 we report the error in the solution for different values of the damping coefficient $\alpha$ and for different grid sizes $N$. The domain is the same ellipse as before, CFL $=0.5$, and smooth start is enabled. For these long time computations, damping is clearly needed, but we see that it suffices to take $\alpha=2 \times 10^{-4}$ to stabilize the scheme for both grid sizes. However, for the finer grid it suffices with $\alpha=1 \times 10^{-4}$. This suggests that $\alpha=h \tilde{\alpha}$, i.e., the damping term can be of the order $\mathcal{O}\left(h^{4}\right)$. Note that there is no apparent increase in the error after long times, which indicates that the damping is very mild and that the scheme is appropriate for long time computations.

\subsection{The $\mathrm{TM}_{z}$ problem}

We proceed by testing our method on the $\mathrm{TM}_{z}$ problem for Maxwell's equations, i.e., the two-dimensional case where $\boldsymbol{H}=H^{(x)}(x, y, t) \boldsymbol{e}_{x}+H^{(y)}(x, y, t) \boldsymbol{e}_{y}$ and $\boldsymbol{E}=E^{(z)}(x, y, t) \boldsymbol{e}_{z}$. By scaling the dependent variables and time, Maxwell's equations describing a homoge- 

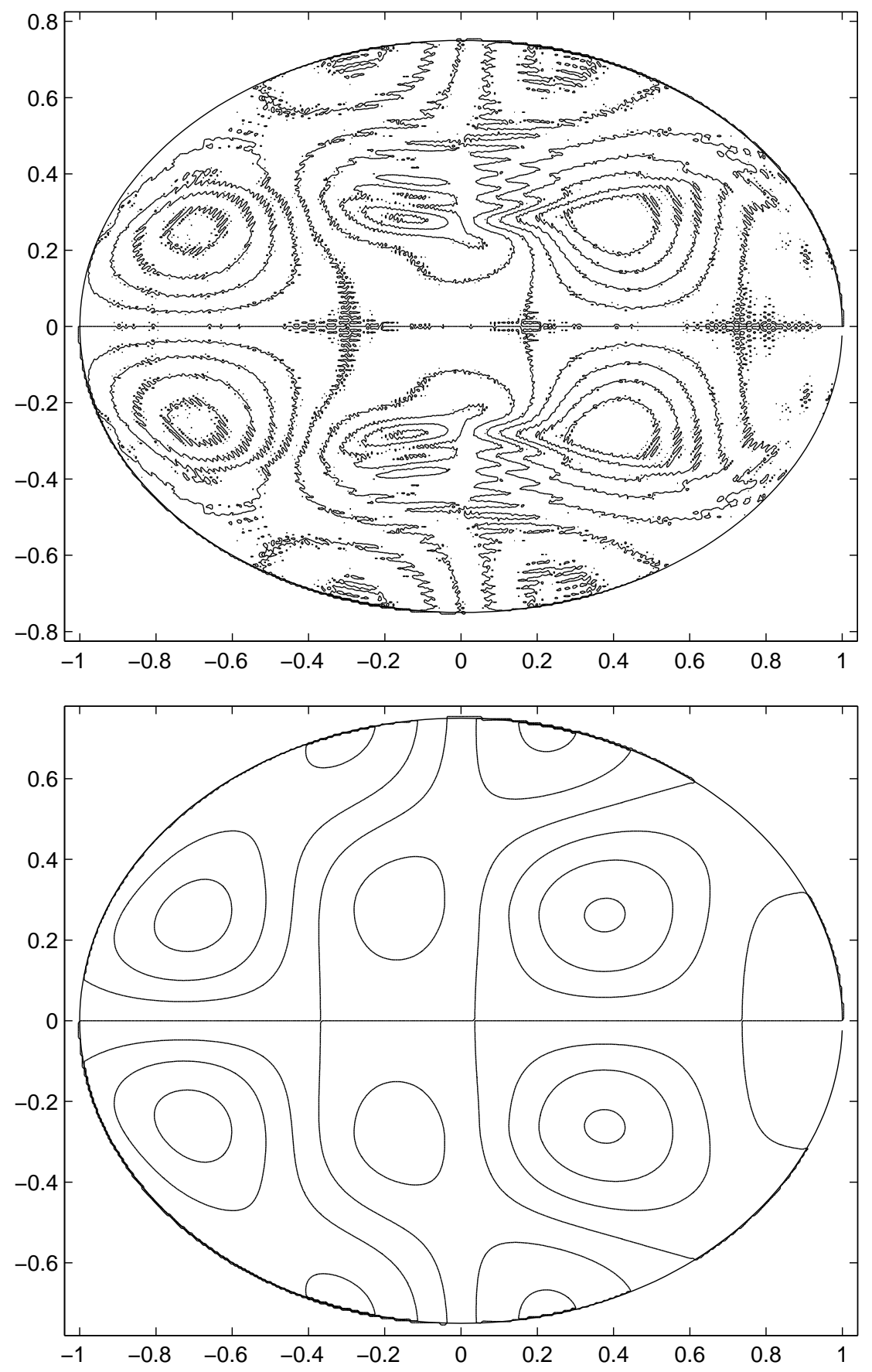

Figure 2: Error in the solution at time $t=2.0$, with direct start (top) and smooth start (bottom). In both cases, the grid size was $h=2.08 / 400(N=401)$ and CFL $=0.5$. Contours are equally spaced in $\left[-4 \times 10^{-4}, 4 \times 10^{-4}\right]$ with step size $10^{-4}$. 


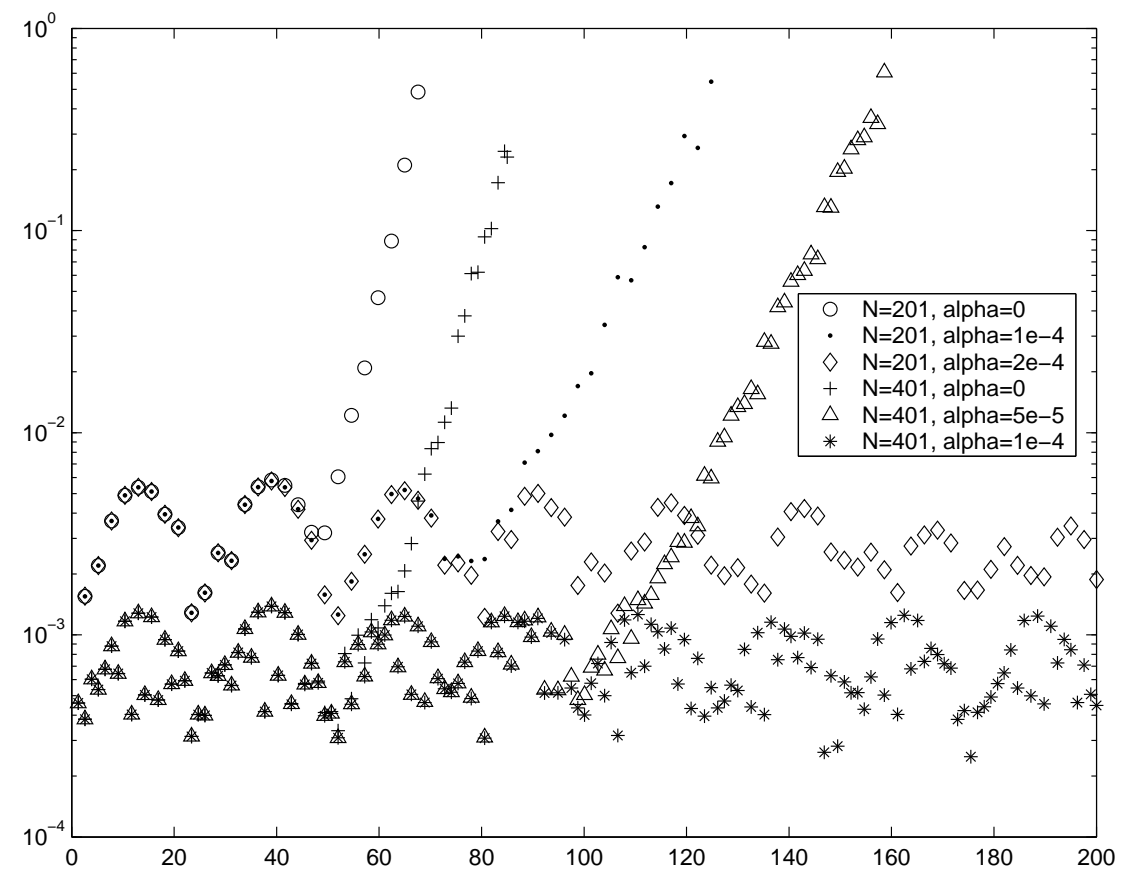

Figure 3: Max error in the solution as function of time for different damping coefficients $\alpha$ and grid sizes $N$.

neous, lossless material without charges simplify to, cf. [11],

$$
\begin{aligned}
\frac{\partial H^{(x)}}{\partial t} & =-\frac{\partial E^{(z)}}{\partial y}, & & \\
\frac{\partial H^{(y)}}{\partial t} & =\frac{\partial E^{(z)}}{\partial x}, & & \text { in } \Omega, \quad t \geq 0, \\
\frac{\partial E^{(z)}}{\partial t} & =\frac{\partial H^{(y)}}{\partial x}-\frac{\partial H^{(x)}}{\partial y}, & &
\end{aligned}
$$

subject to the constraint

$$
\frac{\partial H^{(x)}}{\partial x}+\frac{\partial H^{(y)}}{\partial y}=0, \quad \text { in } \Omega, \quad t \geq 0 .
$$

By cross-differentiation,

$$
\frac{\partial^{2} E^{(z)}}{\partial t^{2}}=\frac{\partial^{2} E^{(z)}}{\partial x^{2}}+\frac{\partial^{2} E^{(z)}}{\partial y^{2}}, \quad \text { in } \Omega, \quad t \geq 0 .
$$

The perfectly electric conducting (PEC) boundary condition $\boldsymbol{n} \times \boldsymbol{E}=0$, where $\boldsymbol{n}=$ $n^{(x)} \boldsymbol{e}_{x}+n^{(y)} \boldsymbol{e}_{y}$ is the outward normal of the boundary, becomes

$$
\left(\boldsymbol{e}_{x} n^{(y)}-\boldsymbol{e}_{y} n^{(x)}\right) E^{(z)}=0, \quad \text { on } \Gamma,
$$


i.e.,

$$
E^{(z)}=0, \quad \text { on } \Gamma .
$$

Instead of directly solving the first order system (53)-(55), we solve the second order equation (57) subject to the Dirichlet boundary condition (58). Once $E^{(z)}$ is computed, the equations for $H^{(x)}$ and $H^{(y)},(53)$ and (54), reduce to ordinary differential equations at each point in $\Omega$.

On the discrete side, (53) and (54) are integrated in time using the second order Adams-Bashforth method,

$$
\begin{aligned}
& H_{i, j}^{(x)}\left(t_{n+1}\right)=H_{i, j}^{(x)}\left(t_{n}\right)-\frac{k}{2}\left(3 D_{0}^{y} E_{i, j}^{(z)}\left(t_{n}\right)-D_{0}^{y} E_{i, j}^{(z)}\left(t_{n-1}\right)\right), \\
& H_{i, j}^{(y)}\left(t_{n+1}\right)=H_{i, j}^{(y)}\left(t_{n}\right)+\frac{k}{2}\left(3 D_{0}^{x} E_{i, j}^{(z)}\left(t_{n}\right)-D_{0}^{x} E_{i, j}^{(z)}\left(t_{n-1}\right)\right),
\end{aligned}
$$

As a result, the following second order accurate centered approximation of the divergence constraint (56) satisfies

$$
D_{0}^{x} H_{i, j}^{(x)}\left(t_{n}\right)+D_{0}^{y} H_{i, j}^{(y)}\left(t_{n}\right)=D_{0}^{x} H_{i, j}^{(x)}(0)+D_{0}^{y} H_{i, j}^{(y)}(0), \quad t_{n}>0 .
$$

Hence, if the discrete divergence is initially zero, it will remain so for all subsequent times.

We use the scheme (15) together with the smooth start procedure to evolve (57). This is a good test of the numerical accuracy of $E^{(z)}$, since $H^{(x)}$ and $H^{(y)}$ depend on the gradient of $E^{(z)}$. Initial conditions for (53)-(55) are $H^{(x)}(\boldsymbol{x}, 0), H^{(y)}(\boldsymbol{x}, 0)$ and $E^{(z)}(\boldsymbol{x}, 0)$. The second order formulation (57) also needs the time derivative of $E^{(z)}$ at $t=0$. This follows from (55),

$$
E_{t}^{(z)}(\boldsymbol{x}, 0)=H_{x}^{(y)}(\boldsymbol{x}, 0)-H_{y}^{(x)}(\boldsymbol{x}, 0) .
$$

Furthermore, to start the Adams-Bashforth scheme (59)-(60), we need an accurate approximation of $E^{(z)}\left(\boldsymbol{x}_{i, j},-k\right)$,

$$
E^{(z)}\left(\boldsymbol{x}_{i, j},-k\right)=E^{(z)}\left(\boldsymbol{x}_{i, j}, 0\right)-k E_{t}^{(z)}\left(\boldsymbol{x}_{i, j}, 0\right)+\frac{k^{2}}{2} \Delta_{h} E^{(z)}\left(\boldsymbol{x}_{i, j}, 0\right) .
$$

When $\Omega$ is a unit circular disc, Maxwell's equations can be solved analytically using separation of variables using polar coordinates $(\rho, \theta)$,

$$
\begin{aligned}
& x(\rho, \theta)=\rho \cos \theta, \\
& y(\rho, \theta)=\rho \sin \theta .
\end{aligned}
$$

We can alternatively express $\boldsymbol{H}$ in terms of its Cartesian or polar components, related by

$$
\begin{aligned}
& H^{(x)}=H^{(\rho)} \cos \theta-H^{(\theta)} \sin \theta, \\
& H^{(y)}=H^{(\rho)} \sin \theta+H^{(\theta)} \cos \theta .
\end{aligned}
$$


Direct start

\begin{tabular}{c|c|c|c|c|c|c}
\hline $\mathrm{N}$ & $\left\|E_{\text {error }}^{(z)}\right\|_{\infty}$ & $\left\|H_{\text {error }}^{(x)}\right\|_{\infty}$ & $\left\|H_{\text {error }}^{(y)}\right\|_{\infty}$ & $\| E_{x}^{(z)}$ error $\|_{\infty}$ & $\| E_{y}^{(z)}$ error $\|_{\infty}$ & $\left\|\Delta E_{\text {error }}^{(z)}\right\|_{\infty}$ \\
\hline 201 & $1.03 \times 10^{-3}$ & $1.47 \times 10^{-3}$ & $1.98 \times 10^{-3}$ & $1.32 \times 10^{-2}$ & $1.63 \times 10^{-2}$ & $8.52 \times 10^{0}$ \\
401 & $2.53 \times 10^{-4}$ & $3.54 \times 10^{-4}$ & $4.90 \times 10^{-4}$ & $3.99 \times 10^{-3}$ & $4.77 \times 10^{-3}$ & $4.02 \times 10^{0}$ \\
\hline \multicolumn{6}{c}{ Smooth start } \\
\hline 201 & $1.59 \times 10^{-3}$ & $3.93 \times 10^{-3}$ & $3.28 \times 10^{-3}$ & $2.61 \times 10^{-3}$ & $5.53 \times 10^{-3}$ & $4.22 \times 10^{-2}$ \\
401 & $3.95 \times 10^{-4}$ & $1.08 \times 10^{-3}$ & $8.41 \times 10^{-4}$ & $6.58 \times 10^{-4}$ & $1.54 \times 10^{-3}$ & $1.06 \times 10^{-2}$ \\
\hline
\end{tabular}

Table 4: Error in the computed solution of the $\mathrm{TM}_{z}$ problem for the mode $m=3, n=1$ at time $t=2.0$.

Let $J_{m}(\xi)$ denote the Bessel function of the first kind of order $m(m=0,1,2, \ldots)$ and let $\mathcal{X}_{m n}$ be the $n$ 'th zero of $J_{m}(n=1,2,3, \ldots)$, i.e., $J_{m}\left(\mathcal{X}_{m n}\right)=0,0<\mathcal{X}_{m 1}<\mathcal{X}_{m 2}<\ldots$ In terms of polar coordinates and components, the $\mathrm{TM}_{z}$ problem is solved by, cf. [11],

$$
\begin{aligned}
& E_{m n}^{(z)}(\rho, \theta)=\mathcal{X}_{m n} J_{m}\left(\mathcal{X}_{m n} \rho\right) \cos (m \theta) \cos \left(\mathcal{X}_{m n} t\right), \\
& H_{m n}^{(\rho)}(\rho, \theta)=\frac{m}{\rho} J_{m}\left(\mathcal{X}_{m n} \rho\right) \sin (m \theta) \sin \left(\mathcal{X}_{m n} t\right), \\
& H_{m n}^{(\theta)}(\rho, \theta)=\mathcal{X}_{m n} J_{m}^{\prime}\left(\mathcal{X}_{m n} \rho\right) \cos (m \theta) \sin \left(\mathcal{X}_{m n} t\right)
\end{aligned}
$$

Since the equations are linear, any linear combination of the above solution is also a solution.

We evaluate the numerical scheme by solving the $\mathrm{TM}_{z}$ problem on a unit circular disc, and compare the numerical solution to the exact analytic solution (61)-(63). We consider the case $m=3, n=1$, where $\mathcal{X}_{31} \approx 6.3801618959$. The problem is discretized on a computational grid with grid size $h=2.08 /(N-1)$ and $\mathrm{CFL}=0.5$. In this computation, the coefficient of the artificial term in the Dirichlet boundary condition is $\gamma=0.2$. No stabilization is necessary for these short time computations, and we set $\alpha=0$. In Table 4 we compare the errors when the computation is started directly (from inhomogeneous initial data) and smoothly. In both cases, the errors in $E^{(z)}, H^{(x)}, H^{(y)}, E_{x}^{(z)}$, and $E_{y}^{(z)}$ are all $\mathcal{O}\left(h^{2}\right)$. However, the errors in $\Delta E^{(z)}$ reveal a highly oscillatory component in $E^{(z)}$ when the computation is started directly. Since $\partial \boldsymbol{H} / \partial t$ depends on the gradient of $E^{(z)}$, it is surprising that the errors in $\boldsymbol{H}$ are smaller when the computation is started directly, since the errors in the gradient of $E^{(z)}$ are smaller when the smooth start procedure is used.

In terms of our scaled variables, the total field energy within $\Omega$ is

$$
E_{\text {field }}(t)=\int_{\Omega} \boldsymbol{E} \cdot \boldsymbol{E}+\boldsymbol{H} \cdot \boldsymbol{H} d \Omega
$$


Maxwell's equations (53)-(55) subject to a homogeneous PEC boundary condition (58) imply $E_{\text {field }}(t)=E_{\text {field }}(0), t>0$. Since our scheme uses damping to ensure stability, it is of interest to evaluate how well the energy (64) is conserved by the numerical approximation.

To evaluate (64), we need to integrate a grid function defined on the embedded boundary grid. Let $g(\boldsymbol{x})$ be a smooth function defined in $\Omega$ and take

$$
g_{i, j}= \begin{cases}g\left(\boldsymbol{x}_{i, j}\right), & \boldsymbol{x}_{i, j} \in \Omega \\ \operatorname{extrap}(g)_{i, j}, & \boldsymbol{x}_{i, j} \notin \Omega, \boldsymbol{x}_{i \pm 1, j} \in \Omega \text { or } \boldsymbol{x}_{i, j \pm 1} \in \Omega, \\ 0, & \text { otherwise, }\end{cases}
$$

where $\operatorname{extrap}(g)_{i, j}$ is defined by linear extrapolation from the interior points. We approximate the integral by a sum of grid cell averages of $g$,

$$
\int_{\Omega} g(\boldsymbol{x}) d \Omega \approx \sum_{i, j} C_{i+1 / 2, j+1 / 2} \frac{g_{i, j}+g_{i+1, j}+g_{i, j+1}+g_{i+1, j+1}}{4} .
$$

For interior cells where all four grid vertices are inside $\Omega$, the cell area is $C_{i+1 / 2, j+1 / 2}=h^{2}$. When all four grid vertices are outside $\Omega$, the cell area is $C_{i+1 / 2, j+1 / 2}=0$. For cells cut by the boundary, we first compute the intersections between the boundary and the four grid lines $x_{i}, x_{i+1}, y_{j}, y_{j+1}$. To estimate the cell area, the cell boundary is approximated by a linear segment between each intersection point. This procedure together with the linear extrapolation to define $\operatorname{extrap}(g)$ in points just outside the boundary has been verified to give a second order accurate quadrature formula.

In Figure 4 we plot the error in the relative energy $E_{\text {field }}(t) / E_{\text {field }}(0)$ as function of time for a circular domain. As before, we study the mode $m=3, n=1$ and use the same parameters as before. For these long time computations, the stabilization term is necessary, and we set $\alpha=10^{-3}$. As can be expected, the conservation error is $\mathcal{O}\left(h^{2}\right)$ and grows approximately linearly in time. For the fine mesh, the relative energy is conserved to within $5 \times 10^{-5}$ at time $t=200$, and we conclude that the effect of the stabilization term is very small.

Next we study the evolution of a pulse centered at $(x, y)=\left(x_{F}, y_{F}\right)$ in a more complicated region where the boundary is represented by a cubic spline, see Figure 5 . We start the computation from a localized perturbation in $E^{(z)}$ near $\left(x_{F}, y_{F}\right)$,

$$
E^{(z)}(x, y, 0)=\phi\left(\sqrt{\left(x-x_{F}\right)^{2}+\left(y-y_{F}\right)^{2}}\right)
$$

To make the perturbation propagate (essentially) radially outward from $\left(x_{F}, y_{F}\right)$, we take

$$
\frac{\partial E^{(z)}}{\partial t}(x, y, 0)=-\phi^{\prime}\left(\sqrt{\left(x-x_{F}\right)^{2}+\left(y-y_{F}\right)^{2}}\right) .
$$




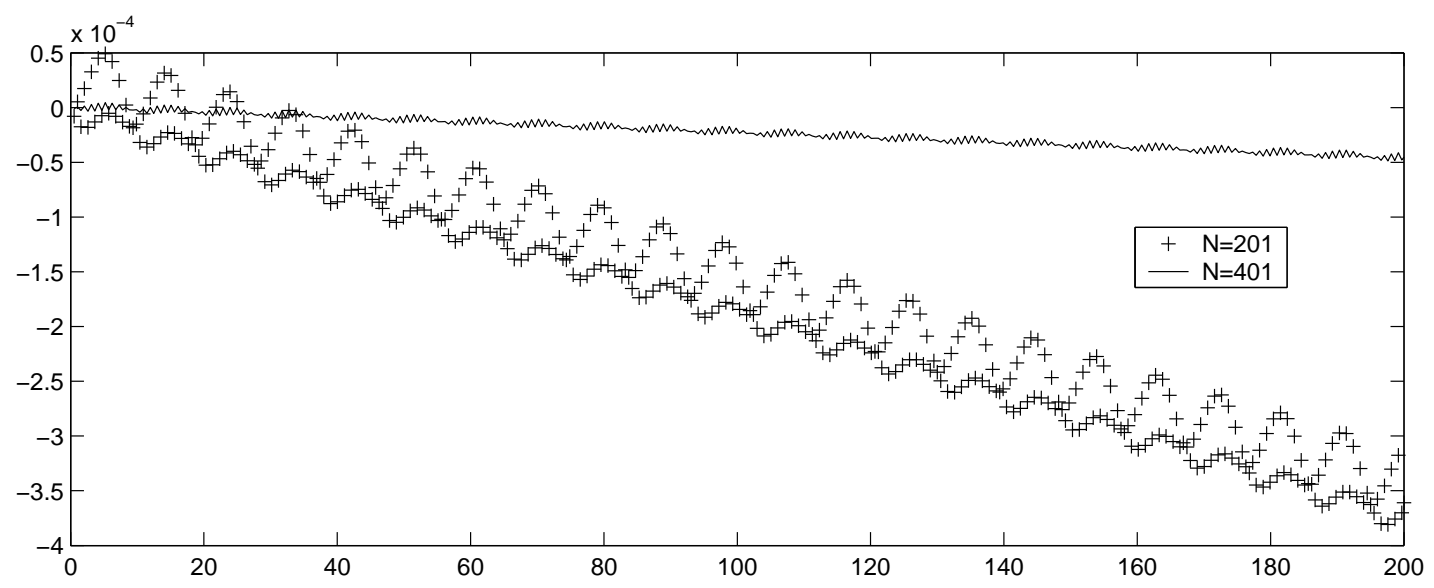

Figure 4: The error in the relative energy $E_{\text {field }}(t) / E_{\text {field }}(0)$ as function of time for a circular domain for the mode $m=3, n=1$ of (61)-(63).

However, to start the computation we must specify compatible initial data for $\left(H^{(x)}, H^{(y)}\right)$. Let $\psi(x, y)$ be a scalar field. The initial $\boldsymbol{H}$ field will be divergence free if

$$
\begin{aligned}
& H^{(x)}(x, y, 0)=-\frac{\partial \psi}{\partial y} \\
& H^{(y)}(x, y, 0)=\frac{\partial \psi}{\partial x}
\end{aligned}
$$

Note that (55) yields

$$
\frac{\partial E^{(z)}}{\partial t}(x, y, 0)=\Delta \psi
$$

Hence, we can find the initial data for $\left(E^{(x)}, E^{(y)}\right)$ by solving the Poisson problem

$$
\Delta \psi=-\phi^{\prime}
$$

and inserting the gradient of $\psi$ in (66)-(67). In special cases, this can be done analytically. Introduce polar coordinates $(\rho, \theta)$ centered around the point $\left(x_{F}, y_{F}\right)$,

$$
\begin{aligned}
& x=x_{F}+\rho \cos \theta, \\
& y=y_{F}+\rho \sin \theta,
\end{aligned}
$$

and let $\phi$ be independent of $\theta$, i.e., $\phi=\phi(\rho)$. The solution of (68) is then

$$
\psi_{\rho}(\rho)=-\phi(\rho)+\frac{1}{\rho} \int_{0}^{\rho} \phi\left(\rho^{\prime}\right) d \rho^{\prime}
$$




\begin{tabular}{c|c|c|c} 
& $X=E^{(z)}$ & $X=H^{(x)}$ & $X=H^{(y)}$ \\
\hline$\left\|X_{h}-X_{4 h}\right\|_{\infty}$ & 0.3636 & 0.3552 & 0.2491 \\
$\left\|X_{h}-X_{2 h}\right\|_{\infty}$ & 0.0744 & 0.0621 & 0.0622 \\
\hline ratio & 4.89 & 5.24 & 4.00 \\
\hline
\end{tabular}

Table 5: Convergence of the discrete solution.

and since $\psi$ is independent of $\theta, \psi_{x}=\left(x-x_{F}\right) \psi_{\rho} / \rho$ and $\psi_{y}=\left(y-y_{F}\right) \psi_{\rho} / \rho$. If we take

$$
\phi(\rho)= \begin{cases}0, & \rho<r_{0}, \\ P\left(\frac{\rho-r_{0}}{r_{1}-r_{0}}\right), & r_{0} \leq \rho \leq r_{1}, \quad, \quad P(\xi)=a \xi^{5}(1-\xi)^{5}(\xi-1 / 2), \\ 0, & r>r_{1} .\end{cases}
$$

we have $\int_{0}^{1} P(\xi) d \xi=0$, so $\psi_{\rho}(\rho)$ has compact support in $r_{0} \leq \rho \leq r_{1}$.

In the computation shown in Figure 5, we used $x_{F}=0.4, y_{F}=0, r_{0}=0.02$ and $r_{1}=0.08$ to give the initial data compact support inside $\Omega$, and set $a=10938.8$ which normalizes the initial data to $-1 \leq E^{(z)}(x, y, 0) \leq 1$. Since the initial data has compact support, the smooth start procedure was not necessary in this computation. No exact solution is known for this problem and we resort to a grid refinement study to access the accuracy of the computed solution. Three calculations were performed where the grid size was $h=5.193 \times 10^{-4}, 2 h$, and $4 h$, respectively. The corresponding grids had $1601 \times 1790$, $801 \times 896$, and $401 \times 448$ grid points, respectively. In this computation, we used $\alpha=10^{-3}$, $\gamma=0.25$ and $\mathrm{CFL}=0.5$. Let $\left(E_{h}^{(z)}, H_{h}^{(x)}, H_{h}^{(y)}\right)$ be the discrete solutions corresponding to grid size $h$. If the discrete solution is resolved on the grid, we expect it to satisfy

$$
E_{h}^{(z)}\left(x_{i}, y_{j}, t_{n}\right)=E^{(z)}\left(x_{i}, y_{j}, t_{n}\right)+h^{2} R\left(x_{i}, y_{j}, t_{n}\right)+\mathcal{O}\left(h^{3}\right)
$$

and similar expressions for $H_{h}^{(x)}$ and $H_{h}^{(y)}$. Hence,

$$
\frac{\left\|E_{h}^{(z)}-E_{4 h}^{(z)}\right\|_{\infty}}{\left\|E_{h}^{(z)}-E_{2 h}^{(z)}\right\|_{\infty}} \rightarrow 5, \quad h \rightarrow 0
$$

and corresponding expressions for $H_{h}^{(x)}$ and $H_{h}^{(y)}$. In Table 5, we report the calculated convergence rates at time $t=0.8$. The calculated rates are near the asymptotic value of 5 which indicates that the solution is second order accurate. The relatively large difference between the solution on the fine and coarse grids indicates that the coarse grid only provides marginal resolution for this problem. 

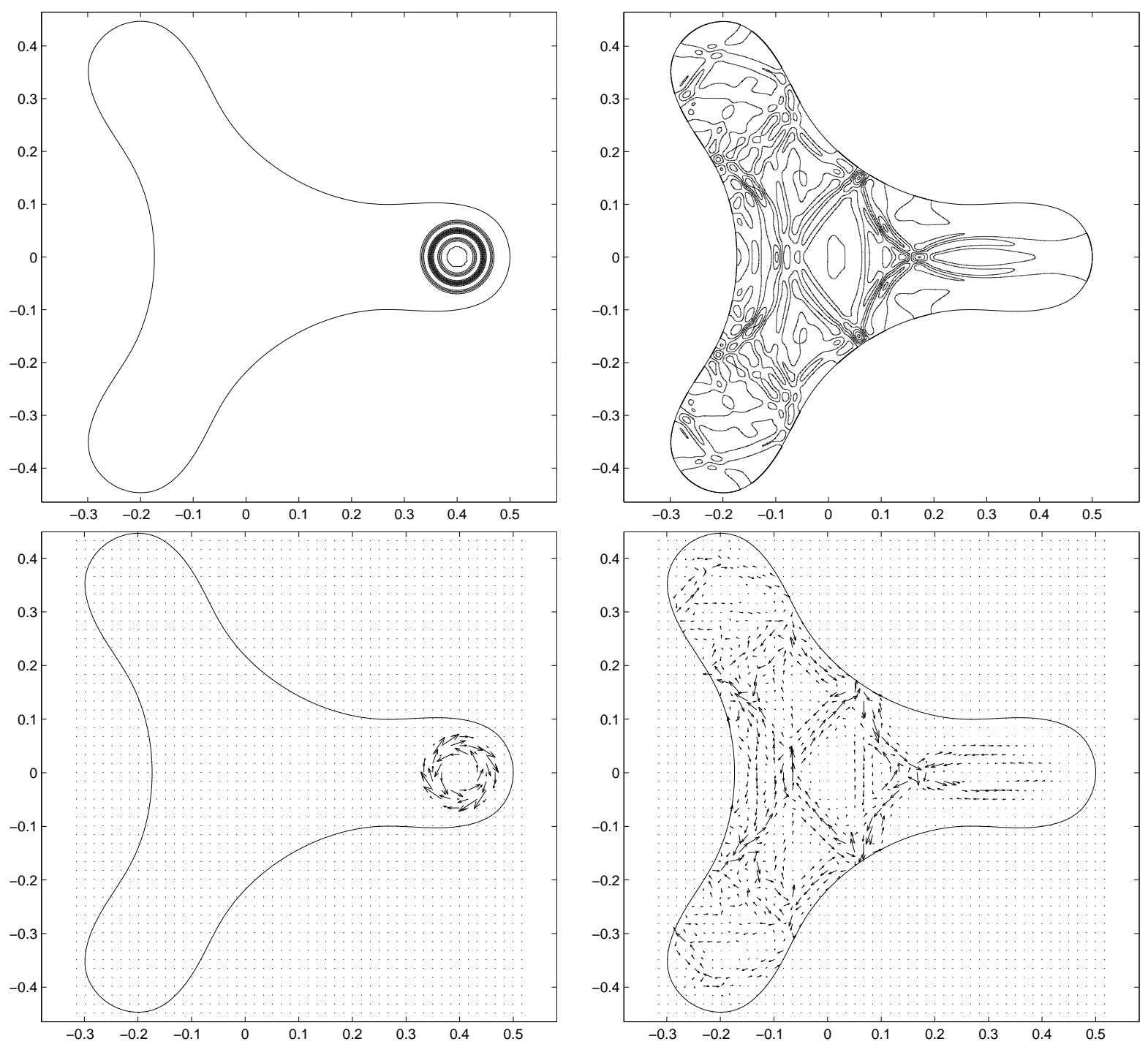

Figure 5: The evolution of $E^{(z)}$ (top) and $\boldsymbol{H}$ (bottom) in the problem for the grid refinement test. The left figures shows initial data and the right figures shows the solution at time $t=0.8$. The solutions are shown on the finest grid with $1601 \times 1790$ grid points. Contours are spaced equally in $[-1.0,1.0]$ with step size 0.2 . Every 32 'nd grid point is used in the vector plots in the bottom row. 


\section{Conclusions}

We have developed a Cartesian embedded boundary method for the second order wave equation in general two-dimensional domains subject to Dirichlet boundary conditions, where both the solution and its gradient are second order accurate. By adding a small artificial term to the discrete boundary condition, we avoid the small-cell stiffness problem and can use an explicit time-integration method where the time step essentially equals that of a periodic domain.

Work is under way to solve Maxwell's equations written as a system of second order wave equations by combining the current method with our previous method for the Neumann problem [2]. Future developments include treatment of discontinuous wave propagation speeds, unbounded domains (far field boundaries), and generalizations to three space dimensions.

\section{A Estimating $\gamma$ in the Dirichlet boundary condition}

Consider the semi-discrete one-dimensional wave equation (23)-(25). After eliminating the ghost point $v_{0}$, we can write the system in matrix form

$$
\boldsymbol{v}_{t t}=A \boldsymbol{v}+\boldsymbol{F}+\boldsymbol{b}, \quad \boldsymbol{v}=\left(v_{1}, v_{2}, v_{3}, \ldots\right)^{T} .
$$

As before, $\boldsymbol{F}$ and $\boldsymbol{b}$ are the interior and boundary forcing terms, respectively. The tridiagonal matrix $A$ satisfies

$$
A=\frac{1}{h^{2}}\left(\begin{array}{ccccc}
-2-\frac{c_{1}-2 \gamma}{c_{0}+\gamma} & 1-\frac{c_{2}+\gamma}{c_{0}+\gamma} & 0 & 0 & \cdots \\
1 & -2 & 1 & 0 & \cdots \\
0 & 1 & -2 & 1 & \\
\vdots & & \ddots & \ddots & \ddots
\end{array}\right) .
$$

Let the eigenvalues of $A$ be $\lambda_{j}$. Since $A$ can be symmetrized by a diagonal matrix, all eigenvalues of $A$ are real valued. For all rows except the first, Gershgorin's circle theorem gives the estimate

$$
\left|h^{2} \lambda_{j}+2\right| \leq 2
$$

that is, $-4 \leq h^{2} \lambda_{j} \leq 0$. The corresponding estimate for the first row is

$$
-3-\frac{c_{1}-c_{2}-3 \gamma}{c_{0}+\gamma} \leq h^{2} \lambda_{j} \leq-1+\frac{\gamma-c_{1}-c_{2}}{c_{0}+\gamma}
$$

We are interested in estimating the eigenvalue with the largest magnitude. Hence, we study how large

$$
q(\alpha, \gamma)=\frac{c_{1}(\alpha)-c_{2}(\alpha)-3 \gamma}{c_{0}(\alpha)+\gamma}
$$


can be for $0 \leq \alpha \leq 1, \gamma>0$. The coefficients in $q$ are given by (22). When $\gamma \leq 0.25$, the maximum of $q$ is attained at $\alpha=0$ and satisfies

$$
\max _{0 \leq \alpha \leq 1} q(\alpha, \gamma)=q(0, \gamma)=\frac{1-3 \gamma}{\gamma}, \quad \gamma \leq 0.25
$$

For $\gamma>0.25, \max q(\alpha, \gamma)<q(0,0.25)$. Hence, to obtain the same lower bound of $\lambda_{j}$ from the first row of $A$ as from the following rows, we require $\max q \leq 1$ and (70) gives $\gamma \geq 0.25$.

\section{References}

[1] H.-O. Kreiss, N. A. Petersson, and J. Yström. Difference approximations for the second order wave equation. SIAM J. Numer. Anal., 40:1940-1967, 2002.

[2] H.-O. Kreiss, N. A. Petersson, and J. Yström. Difference approximations of the Neumann problem for the second order wave equation. UCRL-JC 153184, Lawrence Livermore National Lab, 2003. (to appear in SIAM J. Numer. Anal.).

[3] R. Weller and G. H. Shortley. Calculation of stresses within the boundary of photoelastic models. J. Appl. Mech., 6:A-71-A-78, 1939.

[4] L. Collatz. The numerical treatment of differential equations. Springer verlag, third edition, 1960.

[5] R. B. Pember, J. B. Bell, P. Collella, W. Y. Crutchfield, and M. Welcome. An adaptive Cartesian grid method for unsteady compressible flow in irregular regions. J. Comput. Phys., 120:278, 1995.

[6] M. J. Berger, C. Helzel, and R. J. LeVeque. H-Box methods for the approximation of hyperbolic conservation laws on irregular grids. SIAM J. Numer. Anal., 41:893-918, 2003.

[7] C. Zhang and R. LeVeque. The immersed interface method for acoustic wave equations with discontinuous coefficients. Wave motion, 25:237-263, 1997.

[8] A. Ditkowski, K. Dridi, and J. S. Hesthaven. Convergent Cartesian grid methods for Maxwell's equations in complex geometries. J. Comput. Phys., 170:39-80, 2001.

[9] Hans Johansen and Philip Colella. A Cartesian grid embedded boundary method for Poisson's equation on irregular domains. J. Comput. Phys., 147:60-85, 1998.

[10] D. Gilbarg and N. S. Trudinger. Elliptic Partial Differential Equations of Second Order. Springer Verlag, 1983.

[11] C. A. Balanis. Advanced Engineering Electromagnetics. Wiley, New York, 1989. 